\title{
When psychiatric symptoms reflect medical conditions
}

\author{
Authors: Killian A Welch ${ }^{\mathrm{A}}$ and Alan J Carson ${ }^{\mathrm{A}, \mathrm{B}}$
}

The brain dysfunction associated with certain medical and neurological conditions can produce essentially any psychiatric symptom. This means there is always a chance that presentations thought to be 'psychiatric' are actually explained by unidentified medical pathology. This paper aims to outline an approach to minimise these missed diagnoses.

\section{Introduction}

The days of medical dualism are over. It is beyond rational argument that psychiatric conditions have biological underpinnings. Our understanding of the pathophysiology of, for example, schizophrenia now dwarfs that of many 'medical' conditions, such as migraine. However, it is still the case that psychiatric diagnoses are syndromal, determined by the presence or absence of specific symptoms. With some notable exceptions, diagnostic tests are elusive. Given that many medical and/or neurological disorders can produce mental-state disturbance, the potential for 'medical' conditions to masquerade as psychiatric syndromes remains.

Here, we provide guidance to help clinicians identify when mentalstate disturbance is due to an underlying medical and/or neurological condition. Our focus is on presentations of agitation, emotional disturbance or psychotic symptoms. Guidance for the general physician on somatisation and functional neurological symptoms can be found elsewhere. ${ }^{1,2}$ We do not aim to provide an exhaustive list of medical differential diagnoses. Instead, we have outlined our general approach to such cases, highlighting when clinicians should be especially vigilant to potential underlying medical and/or neurological illness. We also do not discuss the generally poor healthcare received by psychiatric patients. The statistics are shocking; major psychiatric disorder is associated with a 10-15-year reduction in life expectancy, with excess mortality predominantly attributable to medical illness rather than to suicide. ${ }^{3}$ Although the care of patients with mental illness in general hospitals has recently received more attention, ${ }^{4}$ this moral emergency warrants its own paper.

\section{How to avoid misdiagnosis}

Missing medical causes of disturbed mental state can have catastrophic consequences, because the underlying medical

Authors: ${ }^{A}$ consultant neuropsychiatrist, Robert Ferguson Unit, Royal Edinburgh Hospital, Edinburgh, UK; ${ }^{\text {C consultant }}$ neuropsychiatrist, Department of Clinical Neurosciences, Western General Hospital, Edinburgh, UK condition goes untreated. Data on the frequency with which this occurs are limited, but Johnson's case series reporting that $12 \%$ of consecutive psychiatric admissions had some (previously unidentified) physical illness that was judged to be aetiologically important to the presentation remains a salutary lesson. ${ }^{5}$ In the absence of reliable data, we feel that it reasonable to refer to clinical experience, which suggests that the following are crucial in preventing the erroneous attribution of symptoms to psychiatric aetiology.

\section{Think delirium}

In medical inpatients, delirium is the cause of mental-state disturbance until proved otherwise. However, it is often missed and its pleomorphic presentations overlooked completely or mistaken as a psychosis. In its most florid hyperactive form (of which delirium tremens is the archetype), patients are agitated, hallucinating and experiencing persecutory delusions. This can be mistaken for schizophrenia or, because sleep disturbance can be prominent, even mania. However, hypoactive delirium is in fact the more common presentation; these patients often go

\section{Key points}

Mental-state disturbance in medical inpatients is delirium until proved otherwise

Psychiatric conditions tend to develop insidiously rather than over hours to days

A full physical and neurological exam, basic cognitive assessment and routine 'psychiatric' blood screens should be undertaken in all patients presenting with new-onset psychiatric symptoms

Cognitive assessment is fundamental to the identification of delirium and/or encephalopathy, with impairment in tests of sustained attention having particular sensitivity

Limbic encephalitis, especially NMDA receptor antibody encephalitis, should be considered as a differential diagnosis in first presentations of psychosis

KEYWORDS: psychiatry, delirium, encephalitis, psychosis, cognition, diagnosis — 
undetected, or lethargy and psychomotor retardation confused for the avolition and withdrawal of severe depression. ${ }^{6}$

Misdiagnosis can be avoided if it is remembered that delirium is characterised by an abrupt onset, altered conscious level and fluctuating course, features that also distinguish it from dementia. Impaired attention, with associated disorientation, is the key clinical finding. It can be identified through simple bedside tests. At a minimum, one should formally test orientation to time and place and sustained attention, with serial seven subtractions or months of the year backwards being useful tests of the latter. Additional disturbances in cognition (particularly memory, executive and visuospatial functions) are also often present, thinking is muddled, sleep fragmented, and perceptual disturbances, especially illusions and visual hallucinations, can occur. There are many validated screening tools (eg the 4AT, www.the4at.com) and they should be more widely used. Patients with schizophreniform or manic psychosis are generally orientated and have preserved recent memory (Table 1). Although often distractible, they will not have the gross attentional disturbance of delirium. In 'psychiatric' conditions, hallucinations are usually auditory rather than visual. Causes of delirium that are commonly missed are detailed in Table 2. Although it relies on expert interpretation, electroencephalography can be used to distinguish metabolic and other systemic disorders from intracranial pathologies, and provide useful evidence of delirium in challenging cases. ${ }^{7,8}$

The treatment priority in delirium is identifying and addressing precipitants and maintaining factors. Multifactorial causation is the norm, meaning that consideration of potential contributors should continue even after a putative precipitant is identified. The threshold for developing delirium in compromised brains (be it because of age, dementia, multiple sclerosis, Parkinson's disease, or traumatic brain injury) is reduced. If very vulnerable, relevant precipitants (such as sleep disturbance, hunger or simply being in a strange environment) can appear trivial. This undermines the argument 'it can't be delirium because they are not unwell enough'.

\section{Adequate history, neurological exam and cognitive assessment}

Psychiatric symptoms reflect brain dysfunction. When consequent to medical and/or neurological conditions, additional evidence of nervous system dysfunction is likely; this can manifest as motor (eg dysarthria or altered gait), sensory (eg visual field deficits or peripheral neuropathy), cognitive or language disturbance. Given that the ability of the patient to provide a reliable history could be compromised, collateral history should complement the physical, neurological and cognitive examination. This can also identify symptoms that patients are unaware of or reluctant to reveal; examples include the apathy and social inappropriateness accompanying degenerative conditions, such as frontotemporal dementia (FTD), episodes of unresponsiveness with muscle twitching suggestive of complex partial seizures, substance misuse, or the social withdrawal and bizarre preoccupations of a guarded patient developing schizophrenia. Extrapyramidal side effects (ie rigidity or tremor) are common in patients treated with antipsychotic medication, but localising neurological signs are not in keeping with a psychiatric diagnosis and imaging is indicated. Global or focal cognitive deficits might be apparent from patient or informant history, but might only be elicited by formal assessment (eg naming difficulties in semantic dementia or difficulty reading in posterior cortical atrophy). The Addenbrookes
Cognitive Examination Version III provides a brief but impressively comprehensive means to do this, and it (or an equivalent) should be undertaken in all patients in whom cerebral pathology is suspected. It is available online along with guidance for use. ${ }^{9}$ Drug screens are important, but will not detect novel psychoactive substances.

\section{Abnormal findings overlooked}

When medical pathology is missed, case review often highlights clues that were there, but overlooked once it was decided that the presentation was psychiatric. Classic examples are pulmonary embolism attributed to a panic attack or co-occurring psychosis and movement disorder not prompting consideration of Huntington's disease. It is of course the case that patients with psychiatric conditions can have abnormal findings on physical or laboratory investigation that would not justify an 'organic' diagnosis. Examples include sympathetic overactivity and hyperreflexia in anxiety or mildly elevated C-reactive protein (CRP) in depression. It is often prudent to recheck such markers, with reassurance provided when they settle as expected (eg tachycardia normalising when a panic attack resolves).

\section{Be cognisant of how psychiatric disorders present}

Just because a presentation is odd does not make it psychiatric. If in doubt, ask a psychiatrist because they are usually best placed to judge whether a presentation is indeed compatible with a psychiatric syndrome (but see Table 1); useful features include psychiatric conditions generally having an insidious rather than acute onset (although mania can be subacute) and tending to exhibit some consistency in dominant symptoms (rather than the fleeting and changeable symptoms of delirium). Schizophrenia and bipolar affective disorder usually first present in adolescence and/or young adulthood, being less likely explanations for new-onset psychotic symptoms in later life. Psychotic depression can first present at older ages; hallucinations and delusions are mood congruent, and generally focused on guilt, death and decay. Concrete guidance on who to refer to psychiatric and/or liaison psychiatry services is difficult to provide. However, it is the case that, as well as assisting with diagnostic clarity, psychiatric input is also often helpful in advising on management of agitation, risk management, capacity issues, and when use of the mental health act is indicated.

\section{Further assistance in getting it right}

\section{Basic screening}

Although over investigation is to be discouraged, basic screening should be undertaken in all cases of suspected psychiatric illness. As well as mental state, physical, neurological and cognitive exams, this will always include basic laboratory investigates (Table 2). Although scanning of all patients is not justified, ${ }^{10}$ imaging should be undertaken if the presentation is atypical for psychiatric illness or there are other 'red flags' (Table 3). Ordering of other investigations should be determined by individual presentations rather than by protocols, but guidance for investigation of some potential differential diagnoses are detailed in Table 4.

\section{Limbic encephalitis and other recently characterised conditions in which psychiatric symptoms are prominent}

Over the past decade, it has been recognised that various presentations can arise consequent to antibodies directed at 


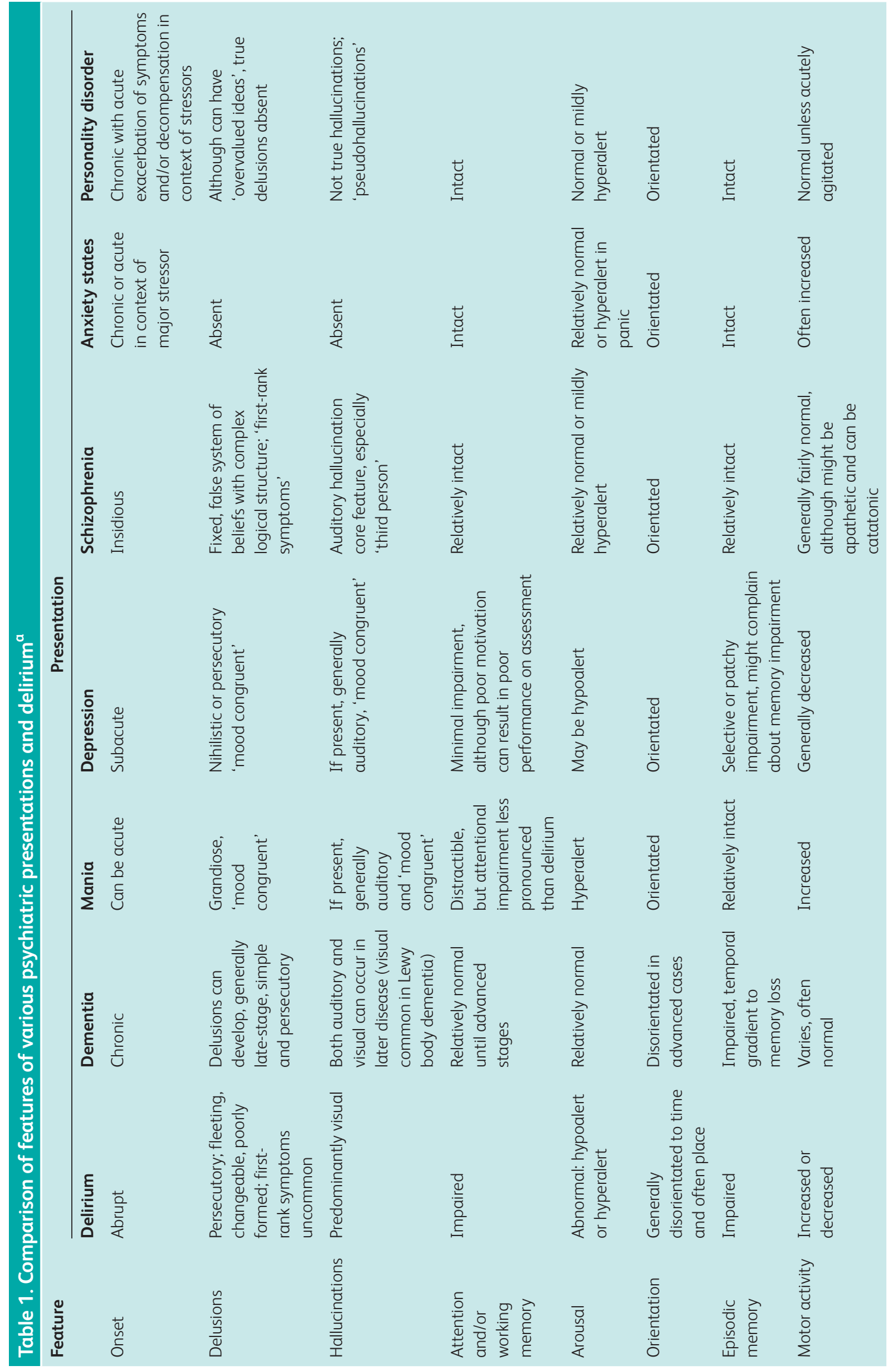




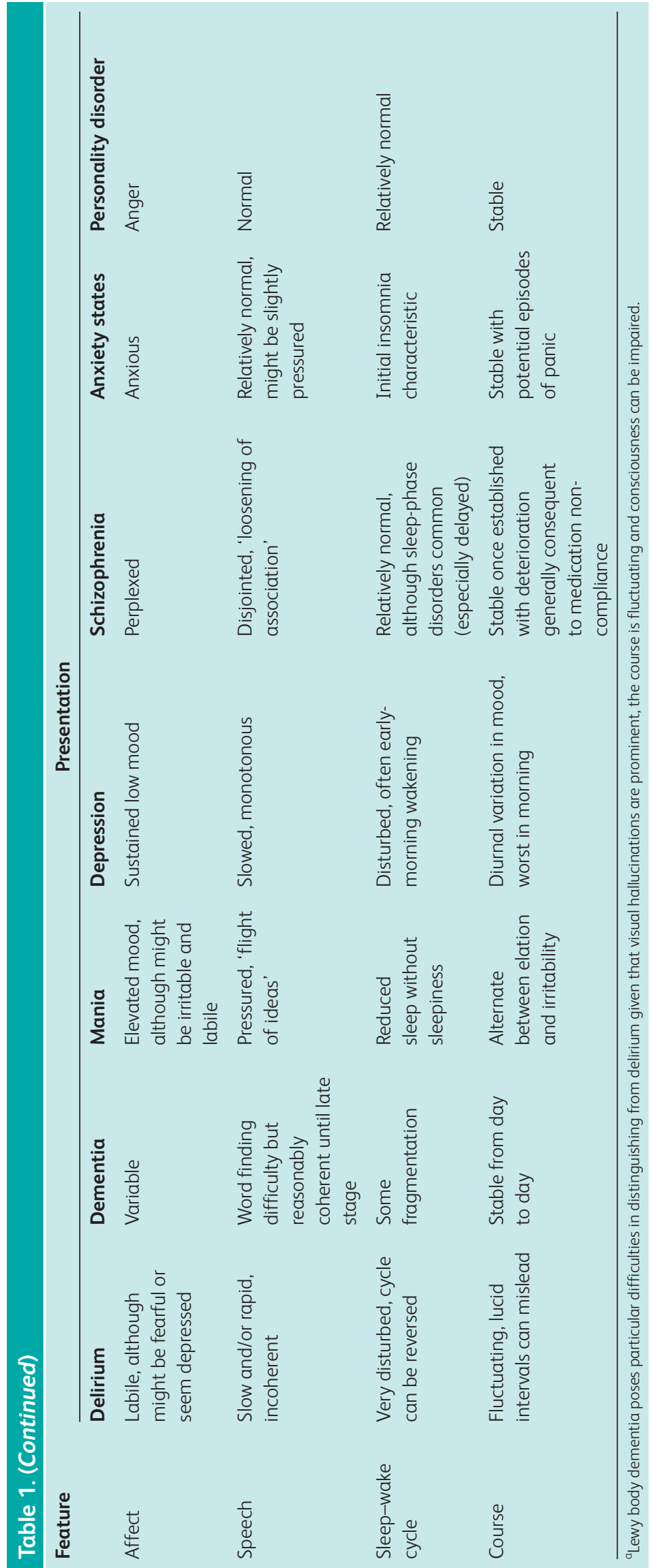


Table 2. Causes of delirium especially likely to be missed

Insult

Modest insults in context of vulnerable brain

Non-convulsive status

Alcohol withdrawal/

delirium tremens

Wernicke's

encephalopathy

Benzodiazepine or other sedative withdrawal

Medication adverse effects

Recreational drug intoxication

Constipation and/or faecal impaction

Sleep deprivation Episodic confusion with sudden onset

\section{Presentation}

Hypoactive, hyperactive or mixed delirium

Hyperactive delirium; sympathetic activation (tachycardia, sweating etc); visual hallucinations

Can occur in absence of alcohol withdrawal; ophthalmoplegia or ataxia might be present

\section{Similar to alcohol withdrawal}

Very common precipitant. Can present as sedated, but delirium can take various forms. Visual hallucinations can be particularly common with anticholinergic drugs

Depends on actions of drug: nystagmus common, stimulants likely associated with sympathomimetic effects and hyperactive delirium

May be no overt symptoms; abdominal pain

History of sleep disorder (eg sleep apnoea)

\section{How to detect}

Core features of delirium; presence of vulnerability factors (eg dementia, brain injury)

EEG; history of epilepsy; vigilance to motor symptoms

Alcohol history; abnormal LFTs/MCV

Characteristic MRI changes (diencephalic hyperintensities on T2-weighted MRI) specific but not sensitive; response to Pabrinex $®$

History of sedative abuse

Be particularly vigilant for medications with anticholinergic effects and opiates

Drug screen, but will not detect novel psychoactive substances; collateral history

Nursing records of bowel movements; abdominal and PR examination; abdominal $\mathrm{X}$-ray

History of disturbed sleep; polysomnography (sleep study)

$\mathrm{EEG}=$ electroencephalography; $\mathrm{LFTs}=$ liver function tests; $\mathrm{MCV}=$ mean corpuscular volume; $\mathrm{MRI}$ = magnetic resonance imaging; $\mathrm{PR}=$ per rectal

neuronal cell surface antibodies that particularly target the limbic system. Although originally identified as paraneoplastic phenomena, it is now recognised that limbic encephalitis can arise in the absence of malignancy, often in young women. Most closely associated with psychiatric presentations is N-methylD-aspartate (NMDA) receptor antibody encephalitis, the early manifestations of which are often anxiety, dramatic expressions of distress (seeming 'hysterical'), affective disturbance and psychosis. Delirium, seizures and severe autonomic disturbance can then supervene, although the range of presentation is wide. Other syndromes relating to other autoantibodies are increasingly recognised (Table 4 ).

These diagnoses should always be considered in the presence of movement disorder, seizures, prominent cognitive impairment, autonomic disturbance or treatment resistance. In many such cases, viral encephalitis will have already been a consideration and have prompted lumbar puncture (LP) and cerebrospinal fluid (CSF) analysis. However, it is conceivable that, in time, screening for these autoimmune conditions will be extended to all first presentations of psychosis. Diagnosis is based on detection of the relevant autoantibodies (presence in CSF being more specific than in serum), imaging (although magnetic resonance imaging [MRI] can be normal) and clinical judgement. Treatment centres on immunosuppression, supportive care and the exclusion and/or identification and treatment of any underlying tumour.

Limbic encephalitis is also a potential differential diagnosis in older first-presentations of psychosis, when an underlying malignancy is more likely. As age increases, the possibility that psychosis is consequent to an underlying neurodegenerative condition also increases. Visual hallucinations can suggest

Table 3. Situations in which brain imaging is mandatory, with magnetic resonance imaging generally being preferred modality

\section{Symptom or sign}

Localising signs on neurological exam

New-onset seizures

Cognitive impairment excessive and/or atypical for psychiatric condition (and not explained by delirium, intoxication, etc)

Possible encephalitis (eg pyrexia, headache, seizures, cognitive impairment)

Possible fall, cognitive impairment, vulnerability factors such as anticoagulation or alcoholism

\section{Why imaging mandatory}

Identify focal pathology and/or exclude space-occupying lesion Identify focal pathology and/or exclude space-occupying lesion Assist in identification of potential neurological or degenerative cause

Visualise inflammation, assist in exclusion of other potential causes of symptoms

Exclude subdural haematoma 
Table 4. Investigation of 'psychiatric' presentations $s^{a, b}$

\section{Indication}

Routine screening for first psychiatric presentations

Localising signs

Cognitive impairment prominent or psychiatric presentation has atypical features (eg age of onset of psychosis, very changeable and/or fluctuating presentation etc); overt neurological symptoms such as movement disorder or seizures

Catatonia: stupor potentially accompanied by negativism, echopraxia, posturing or flexibilitas care (waxy flexibility; a tendency to remain in an immobile posture)

Cognitive impairment, deranged LFTs, movement disorder and grimacing

Clumsiness, weakness, visual changes, speech difficulty and behavioural changes

\section{Investigation}

Full blood count, urea and electrolytes, calcium, phosphate, liver function tests (including GGT), thyroid function tests, ESR, glucose, urine dipstick, drug screen, chest $\mathrm{X}$-ray if respiratory symptoms; BP, pulse, temperature

$B_{12}$ and folate levels

Imaging (CT or MRI)

4-h temperature readings

Imaging, likely MRI

HIV and syphilis serology

\section{Consider EEG}

ANA; if justified clinically also RF, anti-SSA, anti-SSB, $p$-ANCA and c-ANCA

Consider LP and CSF analysis, testing for HSV etc

Serum (and ideally CSF) autoantibodies associated with autoimmune encephalitis: most commonly associated with 'psychiatric' presentation are Ab to NMDA receptor, VGKC receptor complex proteins (LGI1 and CAsPR2), GABAB receptor and AMPA receptor $^{\mathrm{d}}$

MRI

\section{Possibly EEG}

CPK

Ensure metabolic disturbance excluded and consider autoantibody testing etc

Caeruloplasmin; examine for KayserFleischer rings

Establish if immunocompromised (eg organ transplant, corticosteroids, natalizumab)

MRI

CSF testing for JC virus DNA

\section{Rationale}

Exclude obvious causes of delirium, increase chances of detection of relevant general medical condition

Reversible causes of dementia; $B_{12}$ deficiency can present with psychosis

Exclude space-occupying lesion or parenchymal brain damage

Assist detection of infection, especially HSV or other viral encephalitis ${ }^{c}$

Exclude space-occupying lesion, identify generalised or localised atrophy, vascular damage, oedema and/or inflammation etc

Exclude these infectious agents (part of routine screen in some centres)

Identify seizure activity, could help to identify delirium

Detection of CNS vasculitis (ESR can be normal)

If justified on basis of possible of infective or autoimmune encephalitis

Autoimmune encephalitis can present without fever, but psychiatric symptoms can be associated with confusion, memory impairment, movement disorder and/or seizures

Identify focal pathology (especially brainstem, diencephalon) or hydrocephalus

Non-convulsive status

NMS

\section{Detection of Wilson's disease}

Progressive multifocal encephalopathy MRI has characteristic changes 
Table 4. (Continued)

\section{Indication}

Cognitive impairment, apathy, agitation, change in gait and incontinence; occasionally suspiciousness and visual hallucinations

Late-onset psychosis (after 3rd decade) with personality change, disinhibition, executive deficits, semantic memory loss, parkinsonian features or possibly motor weakness

Visual hallucinations, parkinsonian features

Although classically cognitive decline, seizures and stroke-like episodes, there are case reports of presenting as psychosis

Personality change, possibly with psychotic symptoms; restlessness and incoordination evolving into jerky choreiform movements

Fatigue, anxiety, depression, possibly psychosis; weight loss, muscle weakness, light-headedness and hyperpigmentation

Episodic, highly stereotyped symptoms or behaviours with sudden onset and termination

Episodic confusion without obvious cause

Cluster of seizures followed by lucid interval and then florid psychosis, often with grandiosity and religious preoccupations

Episodic delusions, hallucinations, mood disturbance, agitation and/or restlessness; abdominal pain, urinary symptoms, peripheral neuropathy and seizures

Episodes of daytime sleep associated with visual hallucinations

\section{Investigation}

Examination of optic fundi for papilloedema; CT or MRI; CSF tap test (if not contraindicated because of concerns about raised intracranial pressure)

Cognitive assessment

MRI

Genetic testing for C9orf72 and potentially other genetic mutations associated with FTD and/or MND

Dopamine transporter scan

Thyroid peroxidise $\mathrm{Ab}$

Family history; genetic testing

Synacthen test

Collateral history: dysphasia, paresis and motor symptoms

\section{EEG}

Establish diagnosis of epilepsy; EEG to exclude ongoing seizure activity

Family history; urinary testing for porphobilinogen; genetic testing

\section{Rationale}

Consider hydrocephalus

Characteristic deficits of frontotemporal or sematic dementia

Identify regional atrophy in frontal or temporal regions in keeping with bvFTD or SD

Will require discussion with neurologist and/or geneticist and possibly genetic counselling

Exclude Parkinson's disease and/or Lewy body dementia

Consider Hashimoto's encephalopathy; exquisitely steroid responsive encephalopathy

Exclude Huntington's disease

Exclude Addison's disease

Ictal phenomena

Non-convulsive status epilepticus

Post-ictal psychosis

Intermittent porphyria

Multiple sleep latency test; HLA typing; CSF Exclude narcolepsy hypocretin levels

${ }^{a}$ This list of investigations is neither exhaustive nor mandatory; it is intended as loose guidance and to prompt consideration of rarer conditions if suggested by the totality of the presentation. ${ }^{b}$ More-specialist or invasive investigation (eg EEG, LP or genetic testing) will normally be undertaken after discussion with neurologists or other relevant specialists. 'Apyrexial cases of HSV encephalitis are recognised. 'Certain limbic encephalitides are particularly likely to have 'psychiatric' presentations. Classic presentations are as follows: (i) NMDA receptor antibody: female predominance; irritability and insomnia progressing to paranoia, delusions and hallucinations, followed by speech dysfunction, dyskinesias, memory deficits, autonomic instability, and a decrease in the level of consciousness. Seizures can occur at any time during the disease, but tend to occur earlier in males; and (ii) Anti-LGI1 encephalitis (voltage-gated potassium channel antibody group): amnesia and confusion, seizures, movement disorders, sleep disorders; Hyponatraemia and faciobrachial dystonic seizures are particularly associated with both these antibodies. The decision to treat will generally need to be made before antibody test results are available. Autoimmune encephalitis is suggested by subacute onset of memory deficits, psychiatric symptoms or altered consciousness and at least one of new focal CNS findings, seizures not explained by a previously known seizure disorder, CSF pleocytosis (white blood cell count of more than five cells per $\mathrm{mm}^{3}$ ), or MRI features suggestive of encephalitis. ${ }^{12} \mathrm{Ab}=$ antibody; ANA = antinuclear antibody; $\mathrm{p} / \mathrm{c}$-ANCA = perinuclear/cytoplasmic antineutrophil cytoplasmic autoantibodies; $\mathrm{BP}=$ blood pressure; bvFTD = behavioural variant frontotemporal dementia; $C N S=$ central nervous system; $C P K=$ creatine phosphokinase; $C S F=$ cerebrospinal fluid; $C T=$ computed tomography; $E E G$ = electroencephalography; $E S R=$ erythrocyte sedimentation rate; $\mathrm{FTD}$ = frontotemporal dementia; $\mathrm{GGT}=$ Gamma-glutamyltransferase; $\mathrm{HLA}$ = human leukocyte antigen; $\mathrm{HSV}=$ herpes simplex virus; $\mathrm{LP}=$ lumbar puncture; $\mathrm{LFT}$ = liver function test; $\mathrm{MND}$ = motor neurone disease; $\mathrm{MRI}$ = magnetic resonance imaging; $\mathrm{NMS}$ = neuroleptic malignant syndrome; $\mathrm{RF}=$ rheumatoid factor; SSA/B = anti-Sjögren's syndrome A/B 
Lewy body dementia, which, given its fluctuating presentation, is easily mistaken for delirium. However, it is also increasingly recognised that certain genetic mutations giving rise to FTD can initially present with psychosis, which can take the more typically 'psychiatric' form of persecutory delusions and auditory hallucinations. The classic association is with the C9orf72 mutation (which can be tested for); ${ }^{11}$ one would expect poor performance on cognitive tests tapping into executive function (eg letter fluency), a collateral history of disinhibition and personality change, and an MRI scan showing localised frontal atrophy.

\section{Conclusion}

Here, we have focused on cases in which psychiatric symptoms arise from pathology that is already extant and detectable with a reasonable index of suspicion. However, there will always be some cases that only time will reveal; essentially, an underlying pathophysiological process has produced psychiatric symptoms early in its course, before other manifestations. It is often harsh to describe these as 'missed diagnoses', but it does emphasise the importance of a willingness to re-examine diagnoses if presentations change and unexpected symptoms appear. Equally, presentations highly suggestive of an 'organic' basis (rapid onset, disorientation or fluctuating presentation) can occur following psychological distress, sleep deprivation or sensory deprivation in the context of a 'normal' brain. Sometimes, only time and an open mind, on the part of both psychiatrists and physicians, will enable diagnostic clarity.

\section{Declaration of interest}

We confirm that there are no competing interests.

\section{References}

1 Stone J. Functional neurological symptoms. Clin Med 2013;13: 80-3.
2 Chitnis A, Dowrick C, Byng R et al. (2014) Guidance for health professionals on medically unexplained symptoms. London: Royal College of General Practitioners and Royal College of Psychiatrists, 2011.

3 Chang C, Hayes RD, Perera G et al. Life expectancy at birth for people with serious mental illness and other major disorders from a secondary mental health care case register in London. PLOS ONE 2011;6:e19590.

4 Mental Health in General Hospitals: Treat as One, 2017. www. ncepod.org.uk/2017mhgh.html [Accessed 2 December 2017].

5 Johnson DAW. Evaluation of routine physical examination in psychiatric cases. Practitioner 1968;200:686-91.

6 O'Sullivan R, Inouye SK, Meagher D. Delirium and depression: interrelationship and clinical overlap in elderly people. Lancet Psychiatry 2014;1:303-11.

7 Obrecht R, Okhomina FO, Scott DF. Value of EEG in acute confusional states. J Neurol Neurosurg Psychiatry 1979;42:75-7.

8 Van Der Kooi Arendina W, Zaal IJ et al. Delirium detection using EEG: What and how to measure. CHEST J 2015;147:94-101.

9 Stringer S, Hurn J and Burnside AM. Appendix D: Cognitive testing. In: Stringer S, Hurn J and Burnside AM (eds), Psychiatry: breaking the ice: introductions, common tasks and emergencies for trainees. John Wiley \& Sons, Ltd, 2015. http://onlinelibrary.wiley.com/ doi/10.1002/9781118557211.app4/pdf

10 McClellan RL, Eisenberg RL, Giyanani VL. Routine CT screening of psychiatry inpatients. Radiology 1988;169:99-100.

11 Kertesz A, Ang LC, Jesso S et al. Psychosis and hallucinations in frontotemporal dementia with the C9orf72 mutation: A detailed clinical cohort. Cogn Behav Neurol 2013;26:146-54.

12 Graus F, Titulaer MJ, Balu R et al. A clinical approach to diagnosis of autoimmune encephalitis. Lancet Neurol 2016;15:391-404.

Address for correspondence: Dr Killian A Welch, Robert Ferguson Unit, Royal Edinburgh Hospital, Edinburgh EH10 $5 \mathrm{HF}, \mathrm{UK}$.

Email: kwelch1@staffmail.ed.ac.uk 